\title{
Progressive atrophy of pyramidal neuron dendrites in autoimmune MRL-lpr mice
}

\author{
Boris Šakić a, * Henry Szechtman ${ }^{\text {b }}$, Judah A. Denburg ${ }^{\text {c }}$, Grazyna Gorny ${ }^{\text {d,e }}$, Brian Kolb ${ }^{\text {d,e }}$, \\ Ian Q. Whishaw d,e \\ a Department of Psychiatry, HSC 4N4, McMaster University, 1200 Main St. West, Hamilton, Ontario, Canada L8N $3 Z 5$ \\ ${ }^{\mathrm{b}}$ Department of Biomedical Sciences, McMaster University, Hamilton, Ontario, Canada \\ ${ }^{\mathrm{c}}$ Department of Medicine, McMaster University, Hamilton, Ontario, Canada \\ d Department of Psychology and Neuroscience, The University of Lethbridge, Lethbridge, Alberta, Canada \\ ${ }^{\mathrm{e}}$ NeuroDetective Inc., The University of Lethbridge, Lethbridge, Alberta, Canada
}

Received 24 November 1997; revised 13 March 1998; accepted 16 March 1998

\begin{abstract}
The autoimmune-prone MRL-lpr substrain of mice develop an autoimmunity-associated behavioral syndrome (AABS) which resembles in many respects the behavior of animals exposed to chronic stress. The present study examined whether these mice show changes in the morphology of neuronal dendrites, as found in animals exposed to chronic stress. A modified Golgi-Cox procedure was used to visualize the dendrites of pyramidal neurons in the parietal cortex and in the CA1 hippocampal field of 5-week and 14-week old MRL-lpr mice and MRL $+/+$ controls. Reduced dendritic branching and length, and an up to $20 \%$ loss of dendritic spines were observed in parietal and hippocampal pyramidal neurons of MRL-lpr mice at both ages. In the parietal cortex, there was an age-dependent potentiation in the reduction of basilar, but not apical, dendrite branching and length, as well as in the loss of spines on basilar segments. Loss of spines in the hippocampus followed an age-related course for apical but not basilar dendrites. Moreover, compared to age-matched controls, brain weight was smaller in MRL-lpr mice at 14 but not 5 weeks of age. Considering that dendritic atrophy becomes more extensive when autoimmune disease is florid in MRL-lpr mice, it is proposed that immune/inflammatory factor(s) produce dendritic loss. Reduced dendritic complexity may represent, at least in part, a structural basis for the altered behavioral profile of MRL-lpr mice. (C) 1998 Elsevier Science B.V. All rights reserved.
\end{abstract}

Keywords: Autoimmunity; Dendrites; Spines; Inflammation; Hippocampus; Parietal cortex; Lupus; Interleukin-6; Stress; MRL-lpr mice; Behavioral immunology

\section{Introduction}

The hallmark phenomena in inbred MRL/MpJ-lpr (MRL-lpr) and MRL/MpJ $+/+(\mathrm{MRL}+/+)$ murine substrains are hypermaturity, accelerated aging and spontaneous development of a systemic, lupus-like autoimmune disease (Smith and Steinberg, 1983). Although MRL-lpr and MRL $+/+$ mice are similar in many respects (e.g., appearance, size and reproductive age), they differ in the age of onset of autoimmunity. MRL-lpr mice show lupus-

\footnotetext{
* Corresponding author. Tel.: +1 9055259140 ext. 22382; fax: +1 905 5228804; e-mail: sakic@mcmaster.ca
}

like symptoms between the 2nd and the 3rd month of life whereas MRL $+/+$ mice show them several months later (reviewed in the work of Theofilopoulos (1992)). The principal factor accounting for the accelerated autoimmunity in the MRL-lpr substrain is a defect in Fas gene expression, produced by a point mutation in the $l p r$ gene on chromosome 19 (reviewed in the work of Shirai and Klinman (1994)). This mutation interferes with normal Fas-induced apoptosis, and so contributes to prolonged survival of activated lymphocytes and autoreactive T- and B-cell clones.

Coincident with the appearance of serological indices of autoimmunity MRL-lpr mice develop a characteristic be- 
havioral syndrome, which we labeled 'autoimmunity-associated behavioral syndrome' (AABS), a constellation of behavioral alterations found in the MRL-lpr, in contrast to the MRL $+/+$, substrain (Šakić et al., 1997b). This syndrome in MRL-lpr mice resembles behavioral changes induced by chronic, inescapable stress (reviewed in the work of Anisman and Zacharko (1990)), as reflected by diminished exploration of novel objects and space, excessive floating in the forced swim test, reduced responsiveness to sucrose, perseveration on reversal learning, poor active avoidance learning, reduced aggressiveness, and increased 'anxiety' (reviewed in the work of Šakić et al. (1997b) and Szechtman et al. (1997)). Most of the behavioral symptoms appear before overt signs of autoimmune disease, such as generalized lymphadenopathy, dermatitis, joint pathology, or glomerulonephritis. Some behavioral symptoms can be prevented by early immunosuppressive treatment with cyclophosphamide (Šakić et al., 1995, 1996), supporting the suggestion that autoimmune/inflammatory factors induce AABS.

Attempts to identify changes in brain morphology related to AABS failed to identify either cerebral vasculopathic or ischemic lesions in MRL-lpr mice (Hess et al., 1993), as might be expected on the basis of neuropathologic studies of human neuropsychiatric lupus (Johnson and Richardson, 1968; Ellis and Verity, 1979), or the cortical ectopias, as observed in other autoimmune strains (Sherman et al., 1987, 1988, 1990). About 50\% of MRL-lpr mice posses enlarged ventricles (Denenberg et al., 1992), but it is not known whether this is also characteristic of the MRL $+/+$ substrain. The only consistent neuropathologic finding which may account for AABS is the presence of large clusters of $\mathrm{T}$ - and $\mathrm{B}$-cells in the choroid plexus and brain parenchyma of MRL-lpr mice (Alexander et al., 1983; Vogelweid et al., 1991; Hess et al., 1993; Farrell et al., 1997). In light of such findings, and following our notion that AABS resembles the effects of chronic stress (Šakić et al., 1992), we reasoned that structural brain changes in MRL-lpr mice would be of the kind observed after chronic stress. Neuronal dendrites are one of the most plastic structural brain elements, undergoing growth or atrophy in response to such diverse manipulations as stress (Magarinos and McEwen, 1995), learning (Comery et al., 1995), circulating hormones (Magarinos and McEwen, 1995), and lesions (Kolb et al., 1997a) Consequently, in the present study we compared dendritic morphology of diseased MRL-lpr mice and MRL $+/+$ controls at two ages: at 5 weeks of age, when few serologic signs of lupus disease are recognizable; and at 14 weeks of age, when the disease is florid (Šakić et al., 1993, 1994). We expected to see little difference between the substrains at an early age, but dendritic atrophy in MRL-lpr mice at a later age. The sensorimotor parietal cortex and the CA1 field of the hippocampus were chosen for this analysis because behavior-related dendritic changes in these areas had been observed previously (Lolova, 1989; Kolb and Gibb, 1991).

\section{Methods}

\subsection{Animals}

Three-week old ( \pm 3 days) MRL-lpr and MRL $+/+$ males ( $n=6$ mice/substrain) of a similar body weight were purchased from the Jackson Laboratory (Bar Harbor, $\mathrm{ME}$ ) and housed singly, under standard laboratory conditions (light phase: 8 AM-8 PM). Three mice from each substrain were sacrificed at 5 or 14 weeks of age. In one 14-week old MRL-lpr mouse the hippocampal region was not examined due to damage during the fixation/preparation period.

\subsection{Golgi stain and analysis}

Mice were anesthetized with isofluorene and transcardially perfused with $0.9 \%$ saline. The brains were removed and immersed in $20 \mathrm{ml}$ Golgi-Cox solution, which was replaced after 7 days with $30 \%$ sucrose solution. Brains were left for additional 2 weeks before being cut on a vibratome (200 $\mu \mathrm{m}$ sections) and developed using a procedure described previously (Kolb and McClimans, 1986). Layer III pyramidal cells in Ziller's area Par 1 were traced using a camera lucida drawing tube, magnified at $250 \times$, that was attached to the microscope. To be included in the data analysis, the dendritic trees of pyramidal cells had to fulfill following criteria: (a) the cell had to be well impregnated and not obscured with blood vessels, astrocytes, or heavy clusters of dendrites from other cells and (b) the apical and basilar arborizations had to appear to be largely intact and visible in the plane of section. The cells were analyzed by drawing the cells using camera lucida and then counting each branch segment and summarizing by branch order using the procedure of Coleman and Riesen (1968). Branch order was determined for the basilar den-

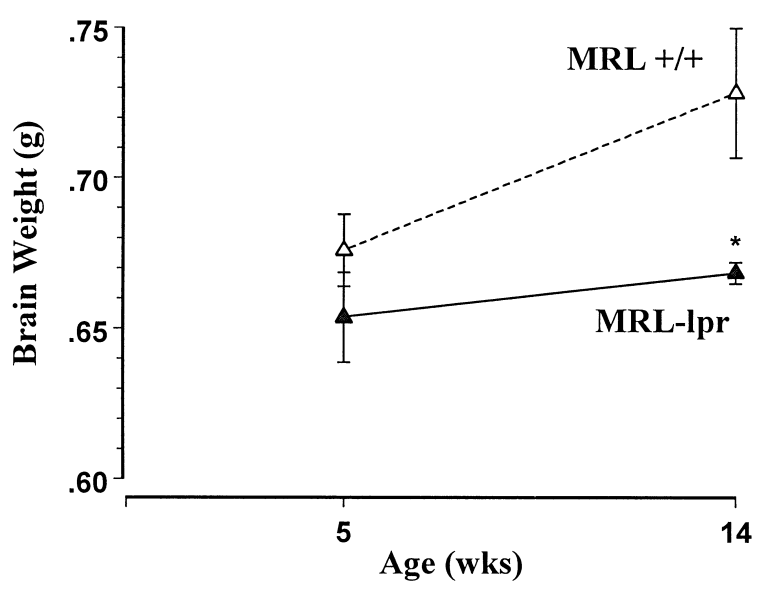

Fig. 1. Wet brain weight of MRL-lpr and MRL $+/+$ control mice at 5 and 14 weeks of age. Each symbol represents the mean \pm SEM of an independent group of animals ( $n=3 /$ group). * Indicates $p<0.05$ compared to MRL $+/+$ group. 
drites such that branches originating at the cell body were first order, after one bifurcation, second order, and so on. Branch order was determined for the apical dendrites such that branches originating from the primary apical dendrite were forst order and so on. Dendritic branching in CAI region was not measured because hippocampal neurons

\section{MRL $-+/+$}
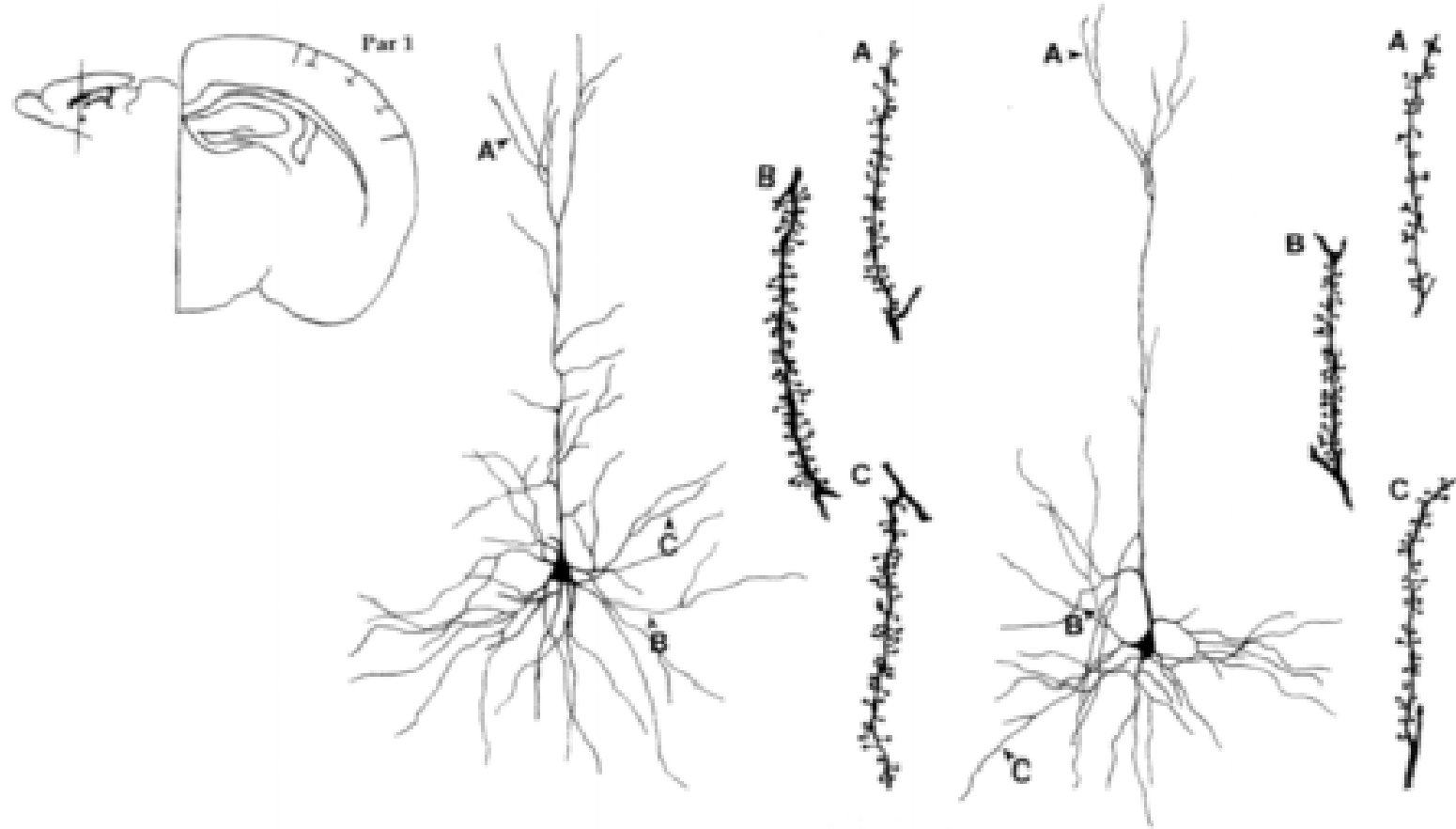

(Inset: a representative photograph of dendrites with deteriorating spines in the MRL-Ipr neuron.)
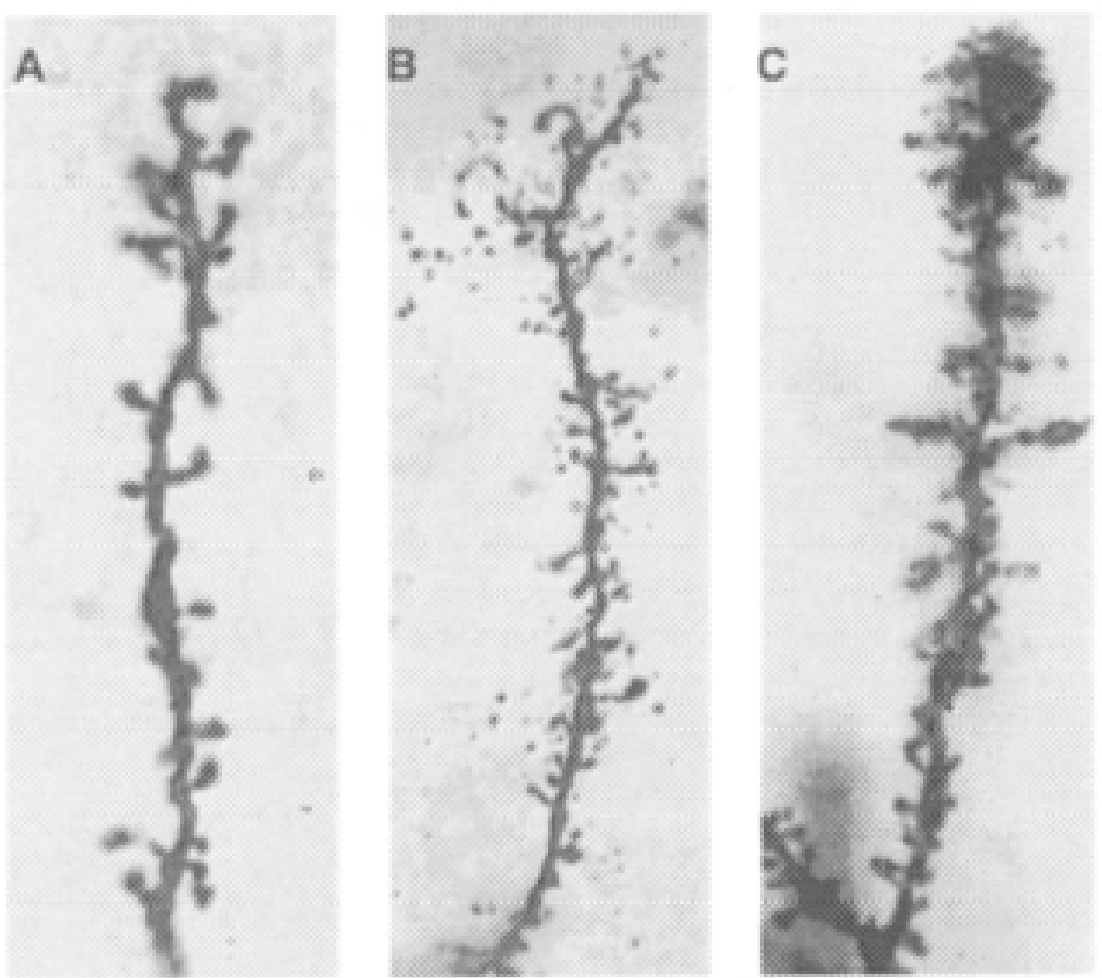

Fig. 2. Drawing of a representative pyramidal neuron from the parietal cortex (Par 1) of a MRL $+/+$ (left) and MRL-lpr mouse (right). Apical (A). basilar medial (B) and basilar terminal dendritic segments (C) are shown with a typical spine distribution. 
reside in families, making it difficult to isolate cells for drawing.

Spine density was measured from one apical dendritic branch in the terminal tuft, from an oblique branch running off the main apical dendritic shaft about halfway up the shaft, and from the secondary branch proximal to the cell body for one basilar branch, following the procedure of Woolley et al. (1990a). Spine density measures were made from a segment greater than $10 \mu \mathrm{m}$ in length. The dendrite was traced at $1000 \times$ using a camera lucida drawing tube, and the exact length of the dendritic segment was calculated. Spine density was expressed as the number of spines per $10 \mu \mathrm{m}$. Because we did not attempt to correct for spines hidden beneath or above the dendritic segment, the spine density values likely underestimated the actual density of the dendritic spines. The following measures were taken: (1) number of branches, crossings and spines in the apical and basilar dendritic segments on pyramidal neurons of the parietal cortex and (2) number of spines on the basilar and apical segments of the hippocampal CA1 neu-

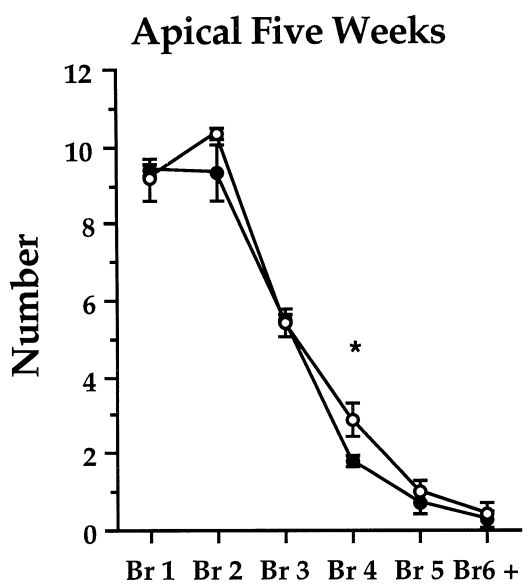

Apical Fourteen Weeks

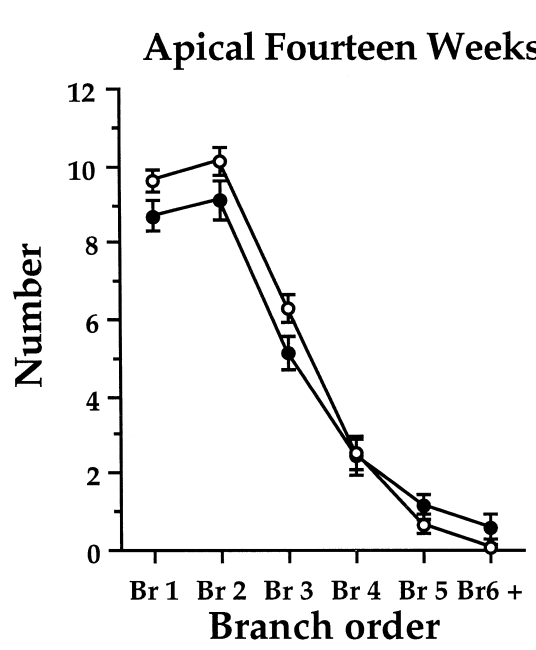

rons. Each measure was obtained independently from the left and the right hemisphere. Five cells in each hemisphere of each mouse were drawn and the computed mean represented a score for a given hemisphere.

\subsection{Indices of autoimmunity}

Urine and blood samples were collected by retro-orbital bleeding under light anesthesia with isofluorine, and were used to measure standard indices of autoimmunity (antinuclear antibody (ANA) titer, hematocrit, proteinuria), as described previously (Šakić et al., 1992). In brief, rat liver was used as a substrate for the indirect immunofluorescent ANA assay. Samples were serially diluted from 1:2 to $1: 16384$, and read by an independent observer. The dilution in the last fluorescent-positive well was considered a measure of antibody concentration and expressed as a $\log _{2}$ value. Relative proteinuria, assessed by colorimetric assay, was coded as follows: trace $=1 ; 30 \mathrm{mg} \%=2 ; 100 \mathrm{mg} \%$ $=3$, and $300 \mathrm{mg} \%=4,2000 \mathrm{mg} \%=5$. Hematocrit was
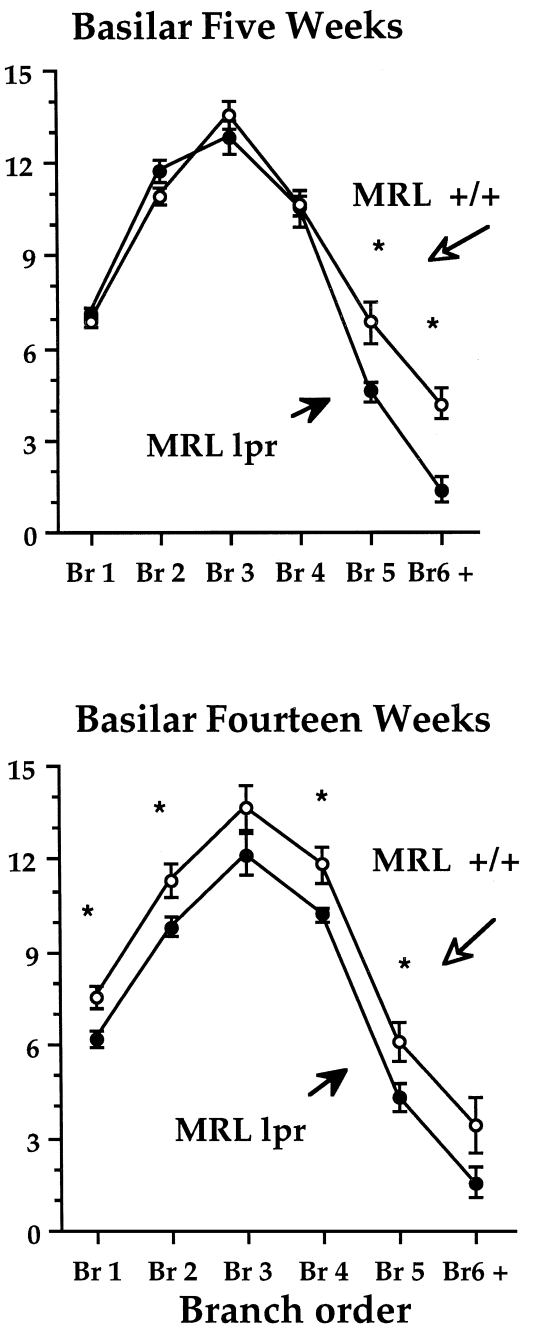

Fig. 3. Number of branches across different orders of apical (left column) and basilar (right column) dendrites in the parietal cortex of 5- and 14-week old MRL-lpr and MRL $+/+$ mice. A significant reduction in the MRL-lpr substrain is indicated by a star. 


\section{Parietal Cortex}

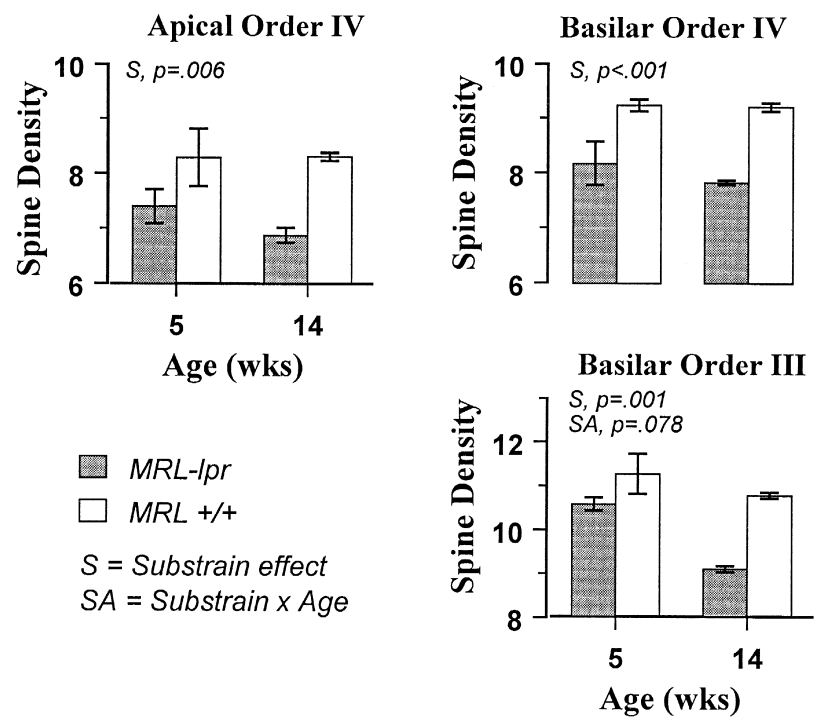

Fig. 4. Measures of spine density on pyramidal neurons in the parietal cortex of MRL-lpr and MRL $+/+$ controls at 5 and 14 weeks of age. Bars are the mean \pm SEM of both hemispheres. Density refers to mean number of spines per $10 \mu \mathrm{m}$ branch.

measured by the Adams microhematocrit method, reflecting indirectly the presence and degree of autoimmune hemolytic anemia in MRL mice.

\subsection{Statistics}

Statistical analysis of morphological measures was performed using a multifactorial ANOVA, with substrain (MRL-lpr vs. MRL $+/+)$ and age (5 vs. 14 weeks) as between group factors, and hemisphere (left vs. right) or branch order as within subjects factors. Student's $t$-tests was used in the post-hoc analysis. A multivariate ANOVA was used to assess the statistical significance of the change in indices of autoimmune disease. Significance level was set at $p<0.05$; trends with $p<0.1$ are also indicated. All computations were performed using the SPSS 7.5 statistical package. Graphs show means \pm SEM.

\section{Results}

As shown in Fig. 1, the brain weight of 14-week old MRL-lpr male mice was significantly lower than that of age-matched MRL $+/+$ controls $(0.668 \pm 0.012$ vs. $0.728 \pm 0.013 \mathrm{~g}, t(4)=3.454, p=0.026)$; brain weight of the younger (5 weeks old) MRL-lpr animals was not statistically different from controls $(0.654 \pm 0.015$ vs. $0.676 \pm 0.022 \mathrm{~g}$ ). In contrast to brain weight, the body weight of MRL-lpr mice was not statistically different from that of MRL $+/+$ males, either at 14 weeks $(47.6 \pm 1.5 \mathrm{~g}$ vs. $49.7 \pm 1.6 \mathrm{~g})$ or 5 weeks of age $(34.2 \pm$ 1.5 vs. $31.9 \pm 1.7 \mathrm{~g}$ ).

The dendritic tree of parietal neurons showed signs of atrophy in MRL-lpr mice (see Fig. 2). As shown quantitatively in Fig. 3, the number of branches along the higher order segments of basilar dendrites were fewer in 5-week old MRL-lpr mice compared to age-matched MRL $+/+$ controls (for substrain by branch order interaction, $F_{6,60}=$ 9.031, $p<0.001)$. At a later age, the reduction was even more pronounced since there were fewer branches along all basilar segments in 14-week old MRL-lpr mice compared to controls (for substrain, $F_{1,10}=23.843, p<0.001$ ). As shown in Fig. 4, spine density on basilar dendrites was also markedly reduced in MRL-lpr mice both along order IV (for substrain, $F_{1,8}=33.66, p<0.001$ ) and order III (for Substrain, $F_{1,8}=22.78, p=0.001$ ) segments.

The reduction in basilar dendritic arbor appeared to be an age-dependent process, as evidenced by a significant substrain by age interaction for number of branches $\left(F_{1,8}\right.$ $=5.36, p=0.049$ ) and dendritic length (Sholl crossings: $F_{1,8}=14.97, p=0.005$; Table 1). There was also a trend for an age-dependent loss in spine density along basilar branch order III segment (for substrain by age interaction, $F_{1,8}=4.07, \quad p=0.078$; Fig. 4) but not along dendritic order IV segment (for substrain by age interaction, $F_{1,8}=$ 0.36 , ns).

Apical dendrites of the parietal cortex neurons were also less arborized in MRL-lpr than MRL $+/+$ males, but the magnitude of this effect was smaller than for the basilar dendrites (number of apical branches: for substrain

Table 1

Summary of the Sholl concentric ring analysis of pyramidal neuron dendritic arbor in the parietal cortex

\begin{tabular}{lll}
\hline Substrain (Age) & Total intersections per neuron (mean \pm SEM) \\
\cline { 2 - 3 } & Apical dendrites & Basilar dendrites \\
\hline MRL-lpr (5 weeks) & $49.7 \pm 1.3$ & $83.7 \pm 1.1$ \\
MRL + / (5 weeks) & $54.3 \pm 1.1$ & $91.7 \pm 0.6$ \\
MRL-lpr (14 weeks) & $47.8 \pm 2.1$ & $73.6 \pm 2.0$ \\
MRL + / (14 weeks) & $50.3 \pm 1.2$ & $93.0 \pm 1.8$ \\
\hline
\end{tabular}

For apical dendrites, the main effect of substrain was significant $(p=0.04)$ and the effect of age was at the borderline of statistical significance $(p=0.078)$. For basilar dendrites, the significant effects were main effects of substrain $(p<0.001)$, Age $(p=0.018)$, and the substrain by age interaction ( $p=0.005)$. Number of Sholl crossings reflects dendritic length. 


\section{CA1 Hippocampus}
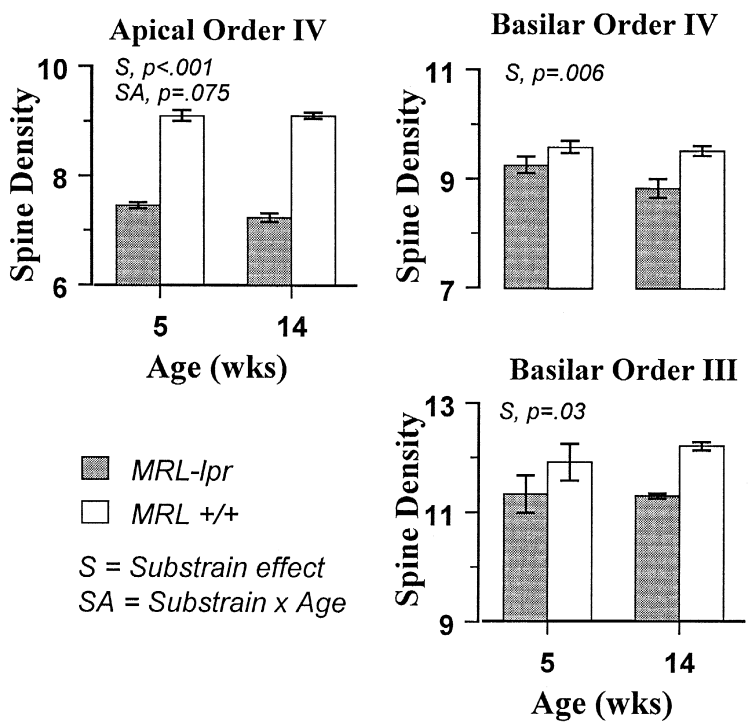

Fig. 5. Measures of spine density on dendrites of pyramidal neurons in CA1 hippocampal field of MRL-lpr and MRL $+/+$ control mice at two ages. Bars are the mean \pm SEM of both hemispheres. Density refers to mean number of spines per $10 \mu \mathrm{m}$ branch.

by branch order interaction: $F_{6,60}=2.251, p=0.05$ at 5 weeks, and $F_{6,60}=2.39, p=0.039$ at 14 weeks, Fig. 3; number of Sholl crossings: for substrain, $F_{1,8}=6.03, p=$
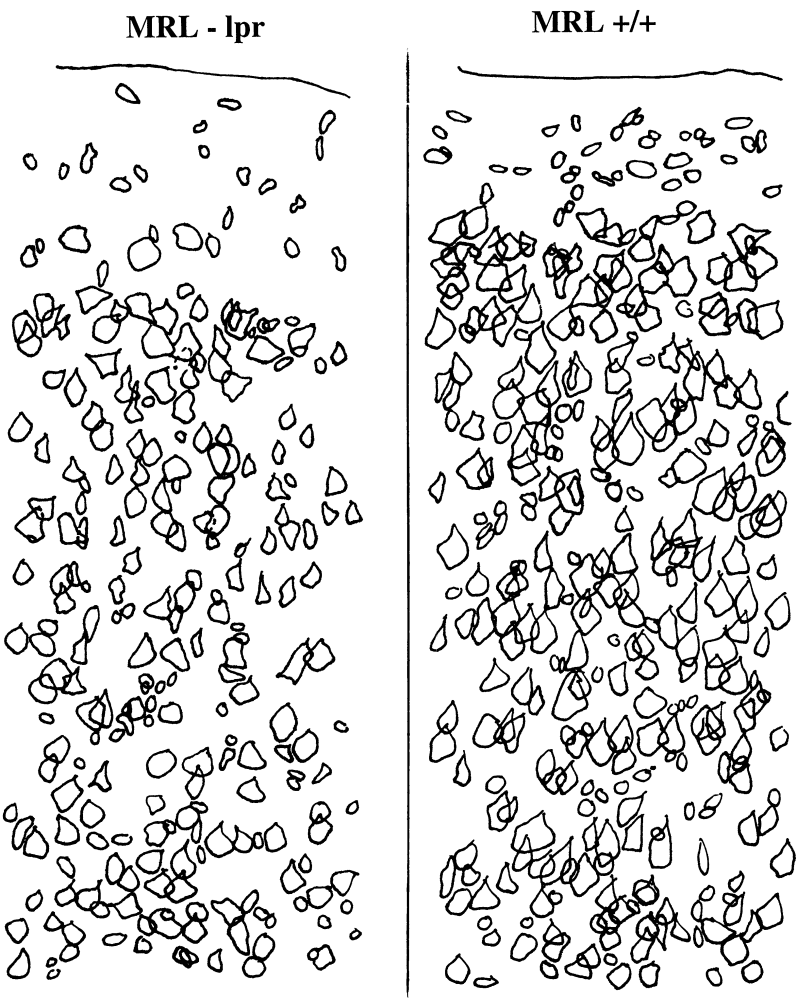

Fig. 6. Illustration of reduced cell density in the parietal cortex of a 14-week old MRL-lpr mouse (left) compared to an age-matched MRL $+/+$ control (right).
Table 2

Indices of systemic autoimmune disease in MRL mice at 5 and 14 weeks of age (mean \pm SEM)

\begin{tabular}{|c|c|c|c|c|}
\hline \multirow[t]{3}{*}{ Measure } & \multicolumn{4}{|l|}{ Group } \\
\hline & \multicolumn{2}{|l|}{ MRL-lpr } & \multicolumn{2}{|c|}{$\mathrm{MRL}+/+$} \\
\hline & 5 weeks & 14 weeks & 5 weeks & 14 weeks \\
\hline ANA titer $\left(\log _{2}\right)$ & $5.0 \pm 0$ & $11.7 \pm 0.7$ & $1.3 \pm 1.3$ & $4.0 \pm 0$ \\
\hline Relative proteinuria & $3.0 \pm 1$ & $4.0 \pm 1.0$ & $2.0 \pm 0$ & $2.0 \pm 1$ \\
\hline Hematocrit (\%) & $49.0 \pm 1$ & $43.0 \pm 1$ & $50.0 \pm 1$ & $50.0 \pm 1$ \\
\hline
\end{tabular}

A multivariate ANOVA showed a significant main effect of substrain $(p=0.003)$ and age $(p=0.001)$, and a significant substrain by age interaction $(p=0.033)$.

0.04, Table 1). The apical dendrites did show however a substantial loss of spines (for substrain, $F_{1,8}=13.68, p=$ 0.006 , Fig. 4). In contrast to basilar dendrite changes, there was no significant substrain by age interaction for any measure of apical dendrite morphology, suggesting that the observed differences between MRL-lpr and MRL $+/+$ controls were not age-related.

In terms of sheer magnitude and robustness, the biggest difference between MRL-lpr and MRL $+/+$ mice in dendritic morphology was present in the CA1 region of the hippocampus. As shown in Fig. 5, the density of spines on apical dendrites in the hippocampus of MRL-lpr mice was $80-83 \%$ of the control density (for substrain, $F_{1,7}=426.7$, $p<0.001$ ) at 14 and 5 weeks of age (for substrain by age, $\left.F_{1,7}=4.37, p=0.075\right)$. MRL-lpr mice had significantly fewer spines also on the basilar pyramidal dendrites in the hippocampus (for substrain: order III segment, $F_{1,7}=7.34$, $p=0.03$; order IV segment, $\left.F_{1,7}=14.88, p=0.006\right)$, but the magnitude of this loss was about $5 \%$.

Although for the present study the number of cortical neurons in the two substrains was not compared quantitatively, nevertheless, visual inspection of the slides (Cresyl violet stain) indicated substantially fewer cells in the parietal cortex of MRL-lpr mice. An example of the apparent cell loss in MRL-lpr mice is shown in Fig. 6.

As shown in Table 2, indices of autoimmunity (presence of serum anti-nuclear antibodies, proteinuria, and lower hematocrit) confirmed that in comparison to MRL $+/+$ congenic controls, MRL-lpr mice suffered from a more severe lupus-like disease (Šakić et al., 1994, 1992).

\section{Discussion}

The present study found a shrinkage of dendritic arborization and loss of dendritic spines in parietal and hippocampal pyramidal neurons of MRL-lpr mice. This atrophy showed relative selectivity for basilar vs. apical dendrites; was relatively large in magnitude; was evident from the earliest age examined; and had an age/diseaserelated course. These four aspects of dendritic atrophy are discussed below in reverse order. 
The number of basilar dendrite branches in the parietal cortex of MRL-lpr mice showed a pattern of atrophy that was statistically greater at 14 than at 5 weeks of age. A similar trend $(p<0.08)$ existed for the density of spines on the terminal segments of basilar dendrites in the cortex and for the density of spines on apical dendrites in the hippocampus. Brain weight of MRL-lpr mice was reduced at 14 but not at 5 weeks of age. It is possible that this pattern reflects a disease-related atrophic process because of the progressive and marked activation of the immune system in MRL-lpr mice from 5 to 14 weeks of age (Šakić et al., 1993). However, the study does not eliminate the possibility that the observed phenomenon reflects an agerelated process of maturation independent of autoimmune/inflammatory factors, and therefore further experiments will be needed to resolve this issue.

Contrary to our expectations, evidence of dendritic atrophy was present in MRL-lpr mice as early as 5 weeks of age, when signs of autoimmune disease are sparse. This finding may indicate an inherent difference in brain morphology between the MRL-lpr and MRL $+/+$ substrains, a lack of dendritic growth factor(s), or an early immuneendocrine imbalance. Specifically, as early as 3 weeks of age and throughout much of their life, MRL-lpr mice exhibit elevated levels of the pro-inflammatory and neuroactive cytokine interleukin-6 (IL-6) (Tang et al., 1991); there is also overexpression of IL-6 mRNA in adult MRLlpr brain (Tsai et al., 1995). Considering neuroactive effects of IL-6 (reviewed in the works of Plata-Salaman (1991) and Schobitz et al. (1994)), and the presence of IL-6 mRNA and IL-6 receptors in the pyramidal layer of the rodent hippocampus and cortex (Schobitz et al., 1993; Gadient and Otten, 1994; Pousset, 1994), we hypothesize that chronic IL-6 production is an early, principal factor which alters brain morphology and behavior (Šakić et al., 1997a). This effect can be mediated either indirectly, via sustained activation of the pituitary-adrenal axis, by some direct effect on brain metabolism/neurotransmission, or both. With regard to the indirect route, it is known that IL-6 induces release of ACTH and corticosteroids (Naitoh et al., 1988; Mastorakos et al., 1993; Stith and Luo, 1994), that the basal levels of serum corticosterone are elevated in MRL-lpr mice (Hu et al., 1993; Lechner et al., 1996), and that chronic exposure to endogenous or exogenous corticosterone produces dendritic atrophy (reviewed in the work of McEwen et al. (1992)). Thus, similarly, an IL-6-driven, chronically 'hyperactive' pituitary-adrenal axis may lead to dendritic atrophy in MRL-lpr mice. With regard to the direct route, chronic exposure of brain cells to IL-6 may result in a cytotoxicity, as suggested by reduced dendritic complexity in transgenic mice overexpressing IL-6 in the brain (Campbell et al., 1993; Steffensen et al., 1994). Although systemic IL-6 does not normally enter the brain, the progression of lupus-like disease is associated with a compromised blood-brain barrier (Hoffman and Harbeck, 1989; Vogelweid et al., 1991). This may facilitate the entry of IL-6 from the periphery and its release from lymphoid cells infiltrated into the choroid plexus (Vogelweid et al., 1991) and brain parenchyma (Farrell et al., 1997). Conceivably, both direct and indirect mechanisms may be operative to different extent at different ages, accounting for the potentiation of the neurodegenerative process in older MRL-lpr mice.

The magnitude of dendritic atrophy in MRL-lpr mice was striking, compared to changes induced by such manipulations as restraint stress, varying hormone levels, or even unilateral devascularizing cortical lesions which result in a $15 \%$ decrease in dendritic branching and spines (Kolb et al., 1997b). In contrast, in the present study, dendritic atrophy in MRL-lpr mice measured as high as $20 \%$. Such loss appears comparable to the changes described for human brains of patients with mental retardation (Jay et al., 1991), Alzheimer's disease (el Hachimi and Foncin, 1990; Einstein et al., 1994), and AIDS (Masliah et al., 1992). Aged rodents show a similar degree of dendritic spine loss in the hippocampus compared to young rats (Lolova, 1989). Reduced dendritic arborization results in synaptic loss, and it has been suggested that cognitive decline in aging and disease is related to degree of synaptic loss (Scheibel et al., 1975; Landfield et al., 1992; Anderson and Rutledge, 1996; Scheff et al., 1997;). Considering that IL-6 is also high in the cerebrospinal fluid of aged humans, and of patients with Alzheimer's disease and AIDS dementia (Perrella et al., 1992b,a; Ershler, 1993; Ershler et al., 1994; Dickson et al., 1996), it is possible that similar ongoing autoimmune/inflammatory process induces dendritic pathology and behavioral changes in these conditions and in autoimmune mice.

Finally, it is noteworthy that an age/disease potentiation of dendritic atrophy in the cortex was selective to basilar over apical dendrites, where there was an acceleration in the reduction of basilar dendrite branching, as well as in the loss of spines on terminal segments. An opposite selectivity was evident in the CA1 region of the hippocampus, where loss of spines followed an age/disease-related course for apical but not basilar dendrites. Select changes in one but not another dendritic region have been observed before (e.g., Woolley et al., 1990b), and are consistent with the notion that the morphologic/functional characteristics of dendrites are not homogenous (Harris and Kater, 1994). Other brain regions of MRL mice will need to be examined, however, to identify the common attributes of the selective atrophy, and in particular whether it represents the loss of specific neural connections, or reflects the topographic distribution of a unique dendritic property, or both.

In summary, the present findings show that in the parietal cortex and the hippocampus of lupus-prone MRLlpr mice, the dendritic complexity of pyramidal neurons is strikingly reduced compared to MRL $+/+$ controls. Such atrophy is evident already at 5 weeks of age but becomes greater at 14 weeks of age, when serologic signs of 
autoimmune disease are florid in MRL-lpr mice. The loss of dendritic complexity and reduced brain weight may represent, at least in part, a morphological basis for the altered behavioral profile of MRL-lpr mice.

\section{Acknowledgements}

B.S. was a post-doctoral fellow of the Ontario Mental Health Foundation. H.S. is a Research Associate of the Ontario Mental Health Foundation. Supported in part by funds from NSERC, Lupus Foundation of Ontario, Lupus Society of Hamilton, and Ontario Lupus Association.

\section{References}

Alexander, E.L., Murphy, E.D., Roths, J.B., Alexander, G.E., 1983. Congenic autoimmune murine models of central nervous system disease in connective tissue disorders. Ann. Neurol. 14, 242-248.

Anderson, B., Rutledge, V., 1996. Age and hemisphere effects on dendritic structure. Brain 119, 1983-1990.

Anisman, H., Zacharko, R.M., 1990. Multiple neurochemical and behavioral consequences of stressors: implications for depression. Pharmacol. Ther. 46, 119-136.

Campbell, I.L., Abraham, C.R., Masliah, E., Kemper, P., Inglis, J.D., Oldstone, M.B., Mucke, L., 1993. Neurologic disease induced in transgenic mice by cerebral overexpression of interleukin-6. Proc. Natl. Acad. Sci. USA 90, 10061-10065.

Coleman, P.D., Riesen, A.H., 1968. Environmental effects on cortical dendritic fields: I. Rearing in the dark. J. Anat. 102, 363-374.

Comery, T.A., Shah, R., Greenough, W.T., 1995. Differential rearing alters spine density on medium-sized spiny neurons in the rat corpus striatum: evidence for association of morphological plasticity with early response gene expression. Neurobiol. Learn. Mem. 63, 217-219.

Denenberg, V.H., Sherman, G.F., Rosen, G.D., Morrison, L., Behan, P.O., Galaburda, A.M., 1992. A behavior profile of the MRL/Mp $1 \mathrm{pr} / \mathrm{lpr}$ mouse and its association with hydrocephalus. Brain Behav. Immun. 6, 40-49.

Dickson, D.W., Lee, S.C., Brosnan, C.F., Sinicropi, S., Vlassara, H., Yen, S.C., 1996. Neuroimmunology of ageing and Alzheimer's disease with emphasis on cytokines. In: Ransohoff, R.M., Benveniste, E.N. (Eds.), Cytokines and the CNS. CRC Press, Boca Raton, FL, pp. 239-267.

Einstein, G., Buranosky, R., Crain, B.J., 1994. Dendritic pathology of granule cells in Alzheimer's disease is unrelated to neuritic plaques. J. Neurosci. 14, 5077-5088.

el Hachimi, K.H., Foncin, J.F., 1990. Loss of dendritic spines in Alzheimer's disease. C.R. Acad. Sci., Ser. III 311, 397-402.

Ellis, S.G., Verity, M.A., 1979. Central nervous system involvement in systemic lupus erythematosus: a review of neuropathologic findings in 57 cases, 1955-1977. Semin. Arthritis Rheum. 8, 212-221.

Ershler, W.B., 1993. Interleukin-6: a cytokine for gerontologists. J. Am. Geriatr. Soc. 41, 176-181.

Ershler, W.B., Sun, W.H., Binkley, N., 1994. The role of interleukin-6 in certain age-related diseases. Drugs and Aging 5, 358-365.

Farrell, M., Šakić, B., Szechtman, H., Denburg, J.A., 1997. Effect of cyclophosphamide on leucocytic infiltration in the brain of MRL/lpr mice. Lupus 6, 268-274.

Gadient, R.A., Otten, U., 1994. Expression of interleukin-6 (IL-6) and interleukin-6 receptor (IL-6R) mRNAs in rat brain during postnatal development. Brain Res. 637, 10-14.

Harris, K.M., Kater, S.B., 1994. Dendritic spines: cellular specializations imparting both stability and flexibility to synaptic function. Ann. Rev. Neurosci. 17, 341-371.

Hess, D.C., Taormina, M., Thompson, J., Sethi, K.D., Diamond, B., Rao, R., Feldman, D.S., 1993. Cognitive and neurologic deficits in the MRL/lpr mouse: a clinicopathologic study. J. Rheumatol. 20, 610617.

Hoffman, S.A., Harbeck, R.J., 1989. CNS lupus and the blood-brain barrier. In: Neuwelt, E.A. (Ed.), Implications of the Blood-Brain Barrier and Its Manipulation. Plenum, New York, pp. 469-494.

Hu, Y., Dietrich, H., Herold, M., Heinrich, P.C., Wick, G., 1993. Disturbed immuno-endocrine communication via the hypothalamopituitary-adrenal axis in autoimmune disease. Int. Arch. Allergy Immunol. 102, 232-241.

Jay, V., Becker, L.E., Chan, F.W., Perry, T.L. Sr., 1991. Puppet-like syndrome of Angelman: a pathologic and neurochemical study. Neurology 41, 416-422.

Johnson, R.T., Richardson, E.P., 1968. The neurological manifestations of systemic lupus erythematosus. Medicine 47, 337-369.

Kolb, B., Gibb, R., 1991. Sparing of function after neonatal frontal lesions correlates with increased cortical dendritic branching: a possible mechanism for the Kennard effect. Behav. Brain Res. 43, 51-56.

Kolb, B., McClimans, J., 1986. Cryostat sectioning of Golgi-Cox tissue. Stain Technol. 61, 379-380.

Kolb, B., Cote, S., Ribeiro-da-Silva, A., Cuello, A.C., 1997a. Nerve growth factor treatment prevents dendritic atrophy and promotes recovery of function after cortical injury. Neuroscience 76, 11391151.

Kolb, B., Forgie, M., Gibb, R., Gorny, G., Rowntree, S., 1997. Age, experience and the changing brain. Neurosci. Biobehav. Rev., in press.

Landfield, P.W., Thibault, O., Mazzanti, M.L., Porter, N.M., Kerr, D.S., 1992. Mechanisms of neuronal death in brain aging and Alzheimer's disease: role of endocrine-mediated calcium dyshomeostasis. J. Neurobiol. 23, 1247-1260.

Lechner, O., Hu, Y., Jafarian-Tehrani, M., Dietrich, H., Schwarz, S., Herold, M., Haour, F., Wick, G., 1996. Disturbed immunoendocrine communication via the hypothalamo-pituitary-adrenal axis in murine lupus. Brain Behav. Immun. 10, 337-350.

Lolova, I., 1989. Dendritic changes in the hippocampus of aged rats. Acta Morpholog. Hung. 37, 3-10.

Magarinos, A.M., McEwen, B.S., 1995. Stress-induced atrophy of apical dendrites of hippocampal CA3c neurons: involvement of glucocorticoid secretion and excitatory amino acid receptors. Neuroscience 69, 89-98.

Masliah, E., Ge, N., Morey, M., DeTeresa, R., Terry, R.D., Wiley, C.A., 1992. Cortical dendritic pathology in human immunodeficiency virus encephalitis. Lab. Invest. 66, 285-291.

Mastorakos, G., Chrousos, G.P., Weber, J.S., 1993. Recombinant interleukin-6 activates the hypothalamic-pituitary-adrenal axis in humans. J. Clin. Endocrinol. Metab. 77, 1690-1694.

McEwen, B.S., Gould, E.A., Sakai, R.R., 1992. The vulnerability of the hippocampus to protective and destructive effects of glucocorticoids in relation to stress. Br. J. Psychiatry 18-23, Suppl.

Naitoh, Y., Fukata, J., Tominaga, T., Nakai, Y., Tamai, S., Mori, K., Imura, H., 1988. Interleukin-6 stimulates the secretion of adrenocorticotropic hormone in conscious, freely-moving rats. Biochem. Biophys. Res. Commun. 155, 1459-1463.

Perrella, O., Carrieri, P.B., Guarnaccia, D., Soscia, M., 1992a. Cerebrospinal fluid cytokines in AIDS dementia complex. J. Neurol. 239, 387-388.

Perrella, O., Guerriero, M., Izzo, E., Soscia, M., Carrieri, P.B., 1992b. Interleukin-6 and granulocyte macrophage-CSF in the cerebrospinal fluid from HIV infected subjects with involvement of the central nervous system. Arq. Neuro-Psiquiatr. 50, 180-182.

Plata-Salaman, C.R., 1991. Immunoregulators in the nervous system. Neurosci. Biobehav. Rev. 15, 185-215. 
Pousset, F., 1994. Developmental expression of cytokine genes in the cortex and hippocampus of the rat central nervous system. Dev. Brain Res. 81, 143-146.

Šakić, B., Szechtman, H., Keffer, M., Talangbayan, H., Stead, R., Denburg, J.A., 1992. A behavioral profile of autoimmune lupus-prone MRL mice. Brain Behav. Immun. 6, 265-285.

Šakić, B., Szechtman, H., Denburg, S.D., Carbotte, R.M., Denburg, J.A., 1993. Spatial learning during the course of autoimmune disease in MRL mice. Behav. Brain Res. 54, 57-66.

Šakić, B., Szechtman, H., Talangbayan, H., Denburg, S.D., Carbotte, R.M., Denburg, J.A., 1994. Behavior and immune status of MRL mice in the postweaning period. Brain Behav. Immun. 8, 1-13.

Šakić, B., Szechtman, H., Denburg, S.D., Denburg, J.A., 1995. Immunosuppressive treatment prevents behavioral deficit in autoimmune MRL-lpr mice. Physiol. Behav. 58, 797-802.

Šakić, B., Denburg, J.A., Denburg, S.D., Szechtman, H., 1996. Blunted sensitivity to sucrose in autoimmune MRL-lpr mice: a curve-shift study. Brain Res. Bull. 41, 305-311.

Šakić, B., Szechtman, H., Braciak, T.A., Richards, C.D., Gauldie, J., Denburg, J.A., 1997a. Reduced preference for sucrose in autoimmune mice: a possible role of interleukin-6. Brain Res. Bull. 44, 155-165.

Šakić, B., Szechtman, H., Denburg, J.A., 1997b. Neurobehavioral alteration in autoimmune mice. Neurosci. Biobehav. Rev. 21, 327-340.

Scheff, S.W., DeKosky, S.T., Price, D.A., 1997. Quantitative assessment of cortical synaptic density in Alzheimer's disease. Neurobiol. Aging $11,29-37$.

Scheibel, M.E., Lindsay, R.D., Tomiyasu, U., Scheibel, A.B., 1975. Progressive dendritic changes in aging human cortex. Exp. Neurol. 47, 392-403.

Schobitz, B., De Kloet, E.R., Sutanto, W., Holsboer, F., 1993. Cellular localization of interleukin- 6 mRNA and interleukin-6 receptor mRNA in rat brain. Eur. J. Neurosci. 5, 1426-1435.

Schobitz, B., De Kloet, E.R., Holsboer, F., 1994. Gene expression and function of interleukin-1, interleukin-6 and tumor necrosis factor in the brain. Prog. Neurobiol. 44, 397-432.

Sherman, G.F., Galaburda, A.M., Behan, P.O., Rosen, G.D., 1987. Neuroanatomical anomalies in autoimmune mice. Acta Neuropathol. Berl. 74, 239-242.
Sherman, G.F., Rosen, G.D., Galaburda, A.M., 1988. Neocortical anomalies in autoimmune mice: a model for the developmental neuropathology seen in the dyslexic brain. Drug Dev. Res. 15, 307-314.

Sherman, G.F., Morrison, L., Rosen, G.D., Behan, P.O., Galaburda, A.M., 1990. Brain abnormalities in immune defective mice. Brain Res. 532, 25-33.

Shirai, A., Klinman, D.M., 1994. The genetic basis of autoimmune disease in MRL-lpr/lpr mice. Int. Rev. Immunol. 11, 179-192.

Smith, H.R., Steinberg, A.D., 1983. Autoimmunity-a perspective. Annu. Rev. Immunol. 1, 175-210.

Steffensen, S.C., Campbell, I.L., Henriksen, S.J., 1994. Site-specific hippocampal pathophysiology due to cerebral overexpression of interleukin-6 in transgenic mice. Brain Res. 652, 149-153.

Stith, R.D., Luo, J., 1994. Endocrine and carbohydrate responses to interleukin-6 in vivo. Circ. Shock 44, 210-215.

Szechtman, H., Šakić, B., Denburg, J.A., 1997. Behavior of MRL mice: an animal model of disturbed behavior in systemic autoimmune disease. Lupus 6, 223-229.

Tang, B., Matsuda, T., Akira, S., Nagata, N., Ikehara, S., Hirano, T., Kishimoto, T., 1991. Age-associated increase in interleukin-6 in MRL/lpr mice. Int. Immunol. 3, 273-278.

Theofilopoulos, A.N., 1992. Murine models of lupus. In: Lahita, R.G. (Ed.), Systemic Lupus Erythematosus. Livingstone, New York, pp. 121-194.

Tsai, C.Y., Wu, T.H., Huang, S.F., Sun, K.H., Hsieh, S.C., Han, S.H., Yu, H.S., Yu, C.L., 1995. Abnormal splenic and thymic IL-4 and TNF-alpha expression in MRL-lpr/lpr mice. Scand. J. Immunol. 41, 157-163.

Vogelweid, C.M., Johnson, G.C., Besch-Williford, C.L., Basler, J., Walker, S.E., 1991. Inflammatory central nervous system disease in lupus-prone MRL/lpr mice: comparative histologic and immunohistochemical findings. J. Neuroimmunol. 35, 89-99.

Woolley, C.S., Gould, E., Frankfurt, M., McEwen, B.S., 1990a. Naturally occurring fluctuation in dendritic spine density on adult hippocampal pyramidal neurons. J. Neurosci. 10, 4035-4039.

Woolley, C.S., Gould, E., McEwen, B.S., 1990b. Exposure to excess glucocorticoids alters dendritic morphology of adult hippocampal pyramidal neurons. Brain Res. 531, 225-231. 